\title{
Candida concentrations determined following concentrated oral rinse culture reflect clinical oral signs
}

Hiroaki Tooyama ${ }^{1}$, Takehisa Matsumoto ${ }^{2 *}$, Kiyonori Hayashi ${ }^{3}$, Kenji Kurashina $^{3}$, Hiroshi Kurita ${ }^{1}$, Mitsuo Uchida ${ }^{4}$, Eriko Kasuga ${ }^{2}$ and Takayuki Honda ${ }^{2}$

\begin{abstract}
Background: Oral candidiasis is an infection caused by a yeast-like fungus called Candida. Various methods can be used to isolate Candida from the oral cavity. However, it is difficult to correctly and satisfactorily diagnose oral candidiasis because currently no microbiological or laboratory standards based on samples from the oral cavity are available. The aim of this study is to establish a reliable laboratory test for diagnosing oral candidiasis.
\end{abstract}

Methods: Oral swab, rinse and concentrated rinse samples were obtained from 200 consecutive outpatients (103 male patients and 97 female patients; mean age, 47.2 years; age range, 9-89 years). Candida colonies from cultured samples were enumerated to compare the sensitivities and specificities of the above sampling methods, and the associations between Candida detection or concentration and the clinical oral signs were examined.

Results: The mean colony numbers were $263 \pm 590 \mathrm{CFU} /$ swab for the swab method, $2894 \pm 6705 \mathrm{CFU} / 100 \mu \mathrm{L}$ for the rinse method, and $9245 \pm 19,030 \mathrm{CFU} / 100 \mu \mathrm{L}$ for the concentrated rinse method. The median numbers were $23 \mathrm{CFU} / \mathrm{swab}$ for the swab method, $56 \mathrm{CFU} / 100 \mu \mathrm{L}$ for the rinse method, and $485 \mathrm{CFU} / 100 \mu \mathrm{L}$ for the concentrated rinse method. Candida was detected in the oral cavity of $33.5 \%$ and $52.0 \%$ of the outpatients by the swab method and concentrated rinse, respectively. Candida concentrations determined by the concentrated rinse were closely related to the severity of the clinical oral signs. The positive predictive values of residual root, redness of the oral mucosa, denture, glossalgia, dry mouth, and taste disorder were useful predictors of oral candidiasis.

Conclusions: Concentrated rinse sampling is suitable for evaluating oral candidiasis, and Candida concentrations examined using this method strongly associated with the oral signs associated with Candida infection.

Keywords: Concentrated oral rinse method, Swab method, Oral candidiasis, Candida

\section{Background}

Oral candidiasis is a common opportunistic infection of the oral cavity and is caused by yeast of the Candida genus, primarily Candida albicans. It presents clinically in many forms, including pseudomembranous (acute/chronic), erythematous (acute/chronic), plaque-like (chronic), and nodular (chronic) forms [1]. However, Candida species are frequently isolated from the oral cavity in healthy individuals of all ages, with a reported prevalence of 15-75\% [2-4], and it is therefore difficult to differentiate oral

\footnotetext{
* Correspondence: ggatcc@shinshu-u.ac.jp

2 Department of Laboratory Medicine, Shinshu University School of Medicine, Shinshu University Hospital, 3-1-1 Asahi, Matsumoto 390-8621, Japan Full list of author information is available at the end of the article
}

candidiasis from the commensal state by microbiological detection of the Candida species in the oral cavity. Furthermore, oral candidiasis has often been diagnosed on the basis of clinical findings, regardless of whether a Candida species was detected. Therefore, additional microbiological criteria are required to diagnose oral Candida infection correctly.

Various methods can be used to isolate Candida from the oral cavity, including smears, plain swabs, imprint cultures, whole saliva collection, concentrated oral rinses, and mucosal biopsies [5, 6]. Of these, the concentrated oral rinse method is one of the most suitable techniques for determining Candida concentrations in the oral cavity [7]; however, this method is inadequate for detecting the 
Candida infection site. Candida concentrations under $600 \mathrm{CFU} / \mathrm{mL}$ in concentrated rinse samples have been reported for healthy commensal carriage [8], whereas individuals with Candida concentrations above 2-3x $10^{3} \mathrm{CFU} / \mathrm{mL}$ are predisposed to oral Candida infection [7]. However, White et al. reported that Candida levels up to $9 \times 10^{3} \mathrm{CFU} / \mathrm{mL}$ were observed in healthy controls and that these levels were occasionally higher than those in patients with oral candidiasis [9].

Oral candidiasis frequently occurs in immunocompromised individuals, including HIV-positive and AIDS patients, organ transplant recipients, and chemotherapy patients [10]. In fact, the disease is often the initial sign of several immunodeficiency diseases, and its clinical significance as a biomarker has been recognized in recent years [11]. However, it is difficult to correctly and satisfactorily diagnose oral candidiasis because currently no microbiological or laboratory standards based on samples from the oral cavity are available. In this study, we examined associations between clinical oral findings and difference methods for obtaining samples from the oral cavity to determine which criteria could help differentiate oral candidiasis from the presence of Candida in the commensal state.

\section{Methods}

Samples obtained from 200 consecutive outpatients (103 male patients and 97 female patients; mean age, 47.2 years; age range, 9-89 years) who consulted a dentist at Aizawa Hospital from March 2011 to June 2011 were participated in this study. Samples from 30 volunteers (17 men and 13 women; mean age, 30.1 years; age range, 23-43 years) without clinical oral symptoms and signs of candidiasis were also used. In all of them, one tooth was not broken and the decayed teeth were completely treated. Informed consent was obtained from all patients, the parents of minors, and volunteers. The Committee for Ethics at Aizawa Hospital approved this study protocol with approval number H22-14.

\section{Sample preparation and determination of CFU}

The 3 sample methods used in the study were as follows.

1. Swab method: The materials were obtained by swabbing the dorsal surface of the tongue with 5 strokes (about $2 \mathrm{~cm}$ in length) of a cotton swab (Hakujuji Co Ltd. Tokyo, Japan), and then the swab was directly inoculated onto CHROMagar Candida medium (Kanto Chemical Co. Ltd., Tokyo, Japan).

2. Rinse method: After a sample had been obtained using the swab method, a sample of oral rinse solution was collected by rinsing the mouth with $10 \mathrm{~mL}$ sterile saline, which was held in the mouth for $5 \mathrm{~s}$ before being collected in a sterile container. One hundred microliters of the rinse solution was inoculated onto the CHROMagar Candida medium.

3. Concentrated rinse method: The oral cavity is rinsed with $10 \mathrm{~mL}$ of sterile saline, and 7 to $10 \mathrm{~mL}$ was collected as the rinse solution. The concentrated rinse solution was prepared by centrifuging it at $2300 \times g$ for $20 \mathrm{~min}$. After the supernatant was removed, the cell pellet was resuspended in $500 \mu \mathrm{L}$, which was inoculated onto CHROMagar Candida medium in $100 \mu \mathrm{L}$ aliquots. Candida colonies were counted after incubation at $37{ }^{\circ} \mathrm{C}$ for $48 \mathrm{~h}$. If there were too many Candida colonies to be counted, the Candida solutions were diluted tenfold.

\section{Associations between the presence of Candida species and clinical oral signs}

We then examined associations between the presence of Candida species and clinical oral signs using samples obtained via the swab method and the concentrated rinse method. Associations between Candida colony counts (Candida concentrations) and clinical oral signs were then determined using samples obtained via the concentrated rinse method. Table 1 shows the clinical oral signs used in this study and their grading.

\section{Oral assessments}

Clinical oral signs were graded as follows. Glossalgia was graded using the Visual Analog Scale (negative: $0 \mathrm{~mm}$; slight: $1 \mathrm{~mm}$; moderate: $30 \mathrm{~mm}$; severe: over $54 \mathrm{~mm}$ ) $[12,13]$. Taste disorder was graded using the Common Terminology Criteria for Adverse Events v3.0 published by the National Cancer Institute (negative: no change in taste; slight: altered taste but no change in diet; moderate: altered taste with change in diet or noxious or unpleasant taste; severe: loss of taste) [14]. Dry mouth was graded using the classification provided by Kakinoki et al. (negative: non-dry; slight: saliva shows viscosity; moderate: saliva showing tiny bubbles on tongue; severe: dry tongue without viscosity, little or no saliva) [15].

Table 1 Clinical oral signs and their grading

\begin{tabular}{lllll}
\hline \multirow{2}{*}{ Signs } & Grade & & & \\
\cline { 2 - 5 } Glossalgia & 0 & 1 & 2 & 3 \\
Taste disorder & Negative & Slight & Moderate & Severe \\
Dry mouth & Negative & Slight & Moderate & Severe \\
Redness of oral mucosa & Negative & Slight & Moderate & Severe \\
Redness of the tongue & Negative & Slight & Moderate & Severe \\
Coated tongue & Negative & Slight & Moderate & Severe \\
Angular cheilitis & Negative & Unilateral & Bilateral & \\
Ulceration & Negative & Single & Multiple & \\
Residual root & Negative & Single & Multiple & \\
Denture & Negative & Unilateral & Bilateral & \\
\hline
\end{tabular}


Redness of oral mucosa was graded using the Eilers Oral Assessment Guide (negative: no redness on the oral mucosa; slight: localized redness areas without ulcerations; moderate: redness on the whole oral mucosa without ulcerations; severe: ulcerations with or without bleeding) [16].

Tongue coating was graded using the visual scores developed by Kojima et al. (negative: less than $1 / 3$ of the tongue slightly coated; slight: about $2 / 3$ of the tongue slightly coated or about $1 / 3$ of the tongue thickly coated; moderate: about $2 / 3$ of the tongue thickly coated; severe: more than $2 / 3$ of the tongue thickly coated [17]. Redness of the tongue was graded similarly (negative: less than $1 / 3$ of the tongue showing slight redness; slight: about $2 / 3$ of the tongue showing slight redness or about $1 / 3$ of the tongue showing strong redness; moderate: about $2 / 3$ of the tongue showing strong redness; severe: more than $2 / 3$ of the tongue showing strong redness).

\section{Determining the normal range of healthy commensal carriage}

We examined 30 volunteers without clinical oral signs of candidiasis for the presence of Candida species. We used the highest colony count obtained from their swab and concentrated rinse samples as the threshold for distinguishing oral candidiasis from the oral commensal state of Candida species. The Candida detection ratio, the associations between clinical oral signs and Candida detection, and the associations between clinical signs and the number of Candida colonies obtained using the swab method and the concentrated rinse method were then determined. The sensitivity and specificity of each clinical sign were examined when Candida species were detected.

\section{Statistical analysis}

The $\chi 2$ test was used to determine the significance of the difference between the rates of positive Candida detection using the oral swab method and the concentrated oral rinse method. The median values of the number of detected Candida which were obtained from identical individuals were compared using the non-parametric Wilcoxon signed rank test. The significance of the relationships between the median Candida concentrations and the grades of each clinical oral sign was analyzed using the nonparametric Kruskal-Wallis test.

Statistical significance was set at $p<0.05$ for all the analysis methods. In addition, Bonferroni test was used to adopt multiple comparison. All statistical analyses were performed using the SPSS software version 22 (SPSS, Chicago, IL, USA).

\section{Results}

In order to establish the required methods before the whole analysis, a pilot test was conducted on the first 10 samples. The colony counts obtained from the first 10 outpatients using the swab, rinse, and concentrated rinse methods are shown in Fig. 1. The median and interquartile range were $23 \mathrm{CFU}$ (interquartile range, 3 to $96 \mathrm{CFU}$ )/ swab for the swab method, $56 \mathrm{CFU}$ (interquartile range, 11 to $900 \mathrm{CFU} / 100 \mu \mathrm{L}$ for the rinse method, and $485 \mathrm{CFU}$ (interquartile range, 210 to $6981 \mathrm{CFU}) / 100 \mu \mathrm{L}$ for the concentrated rinse method in the first 10 outpatients. The first 10 outpatients were tested using all three methods; however, we used the concentrated rinse method for subsequent examinations because it yielded more Candida colonies. The median counts of the Candida colonies obtained using the concentrated rinse method were significantly higher than those obtained using the other two methods, respectively $(p<0.01$, Wilcoxon signed-rank test with Bonferroni test). The concentrated rinse method was the most sensitive, because it could detect Candida species when the swab method or the rinse method did not. Thus, we understood that the concentrated rinse method was appropriate for subsequent examinations.

We presumptively identified Candida species from the color of colonies grown on CHROMagar Candida. Using this method, the following Candida profiles were observed in 68 patients, 12 patients, one patient, nine

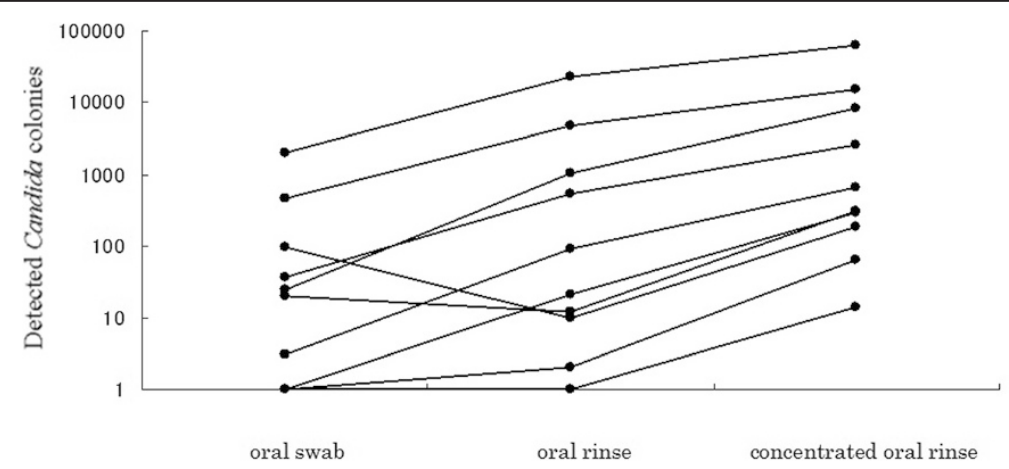

Fig. 1 Comparison of the sensitivities of the swab method, the rinse method, and the concentrated rinse method $(n=10)$. Dots representing data from the same patient are connected by lines 
patients, seven patients, five patients, one patient, and one patient, respectively: C. albicans, C. tropicalis, $C$. glabrata, C. albicans + C. glabrata, C. albicans + C. tropicalis, C. albicans + C. glabrata + C. tropicalis, C. albicans + C. krusei + unidentified Candida species, and $C$. glabrata + C. tropicalis + unidentified Candida species. There were no significant differences in clinical oral signs between the 68 patients with $C$. albicans and the 12 with C. tropicalis.

Detection rates and colony counts obtained using the swab method and the concentrated oral rinse method are shown in Table 2. Candida species were detected in the oral cavity in 67 of 200 patients (33.5\%) by the swab method and in 104 of 200 (52\%) by the concentrated rinse method. The median colony count was $7 \mathrm{CFU}$ (interquartile range, 2 to $37 \mathrm{CFU}$ )/swab for the swab method and 141 CFU (interquartile range, 14 to $1001 \mathrm{CFU}) / 100 \mu \mathrm{L}$ for the concentrated rinse method. The detection ratios $\left(p<0.01, \chi^{2}\right.$ test) and colony counts $(p<0.01$, Wilcoxon signed-rank test) obtained using the concentrated rinse method were significantly higher than those obtained using the swab method.

Associations between clinical oral signs and Candida detection using the swab method are summarized in Tables 3 and 4. When Candida was detected using the swab method, the sensitivities of coated tongue, dry mouth, denture, redness of the tongue, and residual root were $41.8,46.3,40.3,34.3$, and $29.9 \%$, respectively, and the specificities of redness of the oral mucosa, angular cheilitis, residual root, glossalgia, taste disorder, denture, and ulceration were 97.7, 99.2, 92.5, 94.0, 93.2, 88.0, and $92.5 \%$, respectively. The positive predictive values of residual root, redness of the oral mucosa, denture, glossalgia, dry mouth, and taste disorder were 66.7, 66.7, 62.8, $52.9,54.4$, and $35.7 \%$, respectively.

Associations between clinical oral signs and Candida detection using the concentrated rinse method are summarized in Tables 5 and 6. When Candida was detected using the concentrated rinse method, the sensitivities of coated tongue, dry mouth, denture, redness of the tongue, and residual root were 45.2, 42.3, 34.6, 29.8, and $26.0 \%$, respectively, and the specificities of redness of the oral mucosa, angular cheilitis, residual root, glossalgia, taste disorder, denture, and ulceration were 99.0, 99.0, 96.9,
96.9, 95.8, 92.7, and $91.7 \%$, respectively. The positive predictive values of residual root, redness of the oral mucosa, denture, glossalgia, dry mouth, and taste disorder were $90.0,88.9,83.7,82.4,77.2$, and $71.4 \%$, respectively.

Differences between the grades of each clinical oral sign and colony numbers obtained using the swab method are shown in Table 7. High Candida counts were significantly associated with dry mouth. Differences between the grades of each clinical oral sign and colony concentrations obtained using the concentrated rinse method are shown in Table 8. High Candida counts were significantly associated with dry mouth, redness of the tongue, coated tongue, and denture.

When Candida counts were determined in healthy volunteers, the swab method yielded colonies for $3 / 30$ of the volunteers $(1,4$, and 5 colonies, respectively), whereas the concentrated rinse method yielded colonies for 8/30 volunteers $(1,1,2,22,25,36,38$, and 67 colonies, respectively). Based on these results, we defined 0-5 CFU/swab and $0-67 \mathrm{CFU} / 100 \mu \mathrm{L}$ as the reference ranges for healthy commensal carriages detected by the swab method and the concentrated rinse method, respectively. In contrast, among outpatients with no clinical oral signs, the highest counts obtained using the swab method and the concentrated rinse method were $23 \mathrm{CFU} / \mathrm{swab}$ and $90 \mathrm{CFU} /$ $100 \mu \mathrm{L}$, respectively.

\section{Discussion}

In this study, Candida species were detected in the oral cavity in dental clinic outpatients with a frequency of 52.0 and $33.5 \%$ using the concentrated rinse method and the swab method, respectively. Therefore, the concentrated rinse method was more sensitive than the swab method for detecting Candida species in the oral cavity. Some of the oral clinical signs (e.g., coated tongue, dry mouth, denture, redness of the tongue, and residual root) were relatively robust predictors for oral candidiasis. However, the positive predictive values of residual root, redness of the oral mucosa, denture, glossalgia, dry mouth, and taste disorder were high, and only these clinical oral signs were frequently associated with the presence of Candida species.

The concentrated rinse method is more suitable for the detection of Candida species in the oral cavity than

Table 2 Detection rates and colony counts for the swab and concentrated rinse samples from patients $(n=200)$

\begin{tabular}{|c|c|c|}
\hline Sampling method & Positive rate $(\%)$ & $\begin{array}{l}\text { Detected number of Candida } \\
\text { (Median, interquartile range, } \mathrm{CFU} \text { ) }\end{array}$ \\
\hline Oral swab & 33.5 & $7,2-37,(n=67)$ \\
\hline Concentrated oral rinse & 52.0 & $141,14-1001,(n=104)$ \\
\hline
\end{tabular}


Table 3 Indices of clinical oral signs and detection of Candida by the swab method

Association between clinical oral signs and detection of Candida by the swab method

\begin{tabular}{|c|c|c|c|c|}
\hline \multirow[b]{2}{*}{ Clinical oral signs } & & \multicolumn{2}{|c|}{ Candida } & \multirow{2}{*}{$\begin{array}{l}P \text {-value } \\
\left(X^{2} \text { test }\right)\end{array}$} \\
\hline & & $(+)$ & $(-)$ & \\
\hline \multirow[t]{2}{*}{ Glossalgia } & $(+)$ & 9 & 8 & 0.08 \\
\hline & $(-)$ & 58 & 125 & \\
\hline \multirow[t]{2}{*}{ Taste disorder } & $(+)$ & 5 & 9 & 0.85 \\
\hline & $(-)$ & 62 & 124 & \\
\hline \multirow[t]{2}{*}{ Dry mouth } & $(+)$ & 31 & 26 & $<0.01$ \\
\hline & $(-)$ & 36 & 107 & \\
\hline \multirow[t]{2}{*}{ Redness of oral mucosa } & $(+)$ & 6 & 3 & $<0.05$ \\
\hline & $(-)$ & 61 & 130 & \\
\hline \multirow[t]{2}{*}{ Redness of the tongue } & $(+)$ & 23 & 25 & $<0.05$ \\
\hline & $(-)$ & 44 & 108 & \\
\hline \multirow[t]{2}{*}{ Coated tongue } & $(+)$ & 28 & 42 & 0.15 \\
\hline & $(-)$ & 39 & 91 & \\
\hline \multirow[t]{2}{*}{ Angular cheilitis } & $(+)$ & 2 & 1 & 0.22 \\
\hline & $(-)$ & 65 & 132 & \\
\hline \multirow[t]{2}{*}{ Ulceration } & $(+)$ & 5 & 10 & 0.98 \\
\hline & $(-)$ & 62 & 123 & \\
\hline \multirow[t]{2}{*}{ Residual root } & $(+)$ & 20 & 10 & $<0.01$ \\
\hline & $(-)$ & 47 & 123 & \\
\hline \multirow[t]{2}{*}{ Denture } & $(+)$ & 27 & 16 & $<0.01$ \\
\hline & $(-)$ & 40 & 117 & \\
\hline
\end{tabular}

the swab method. However, the number of colonies in the concentrated rinse samples was smaller than the theoretically predicted value of a 20 -fold increase in the rinse samples. This might be related to the low centrifugal force of $2300 \times g$. In addition, the concentrated rinse

Table 4 Indices of clinical oral signs and detection of Candida by the swab method

\begin{tabular}{llll}
\hline \multicolumn{4}{l}{$\begin{array}{l}\text { Sensitivity, specificity, and positive predictive value between clinical oral } \\
\text { signs and detection of Candida by the swab method }\end{array}$} \\
\hline Clinical oral signs & Sensitivity & Specificity & Positive predictive value \\
\hline Glossalgia & $13.4 \%$ & $94.0 \%$ & $52.9 \%$ \\
Taste disorder & $7.5 \%$ & $93.2 \%$ & $35.7 \%$ \\
Dry mouth & $46.3 \%$ & $80.5 \%$ & $54.4 \%$ \\
Redness of oral mucosa & $9.0 \%$ & $97.7 \%$ & $66.7 \%$ \\
Redness of the tongue & $34.3 \%$ & $81.2 \%$ & $47.9 \%$ \\
Coated tongue & $41.8 \%$ & $68.4 \%$ & $40.0 \%$ \\
Angular cheilitis & $3.0 \%$ & $99.2 \%$ & $66.7 \%$ \\
Ulceration & $7.5 \%$ & $92.5 \%$ & $33.3 \%$ \\
Residual root & $29.9 \%$ & $92.5 \%$ & $66.7 \%$ \\
Denture & 40.3 & $88.0 \%$ & $62.8 \%$ \\
\hline
\end{tabular}

Table $\mathbf{5}$ Indices of clinical oral signs and detection of Candida by the concentrated rinse method

Association between clinical oral signs and detection of Candida by the concentrated rinse method

\begin{tabular}{|c|c|c|c|c|}
\hline \multirow[b]{2}{*}{ Clinical oral signs } & & \multicolumn{2}{|c|}{ Candida } & \multirow{2}{*}{$\begin{array}{l}P \text {-value } \\
\left(X^{2} \text { test }\right)\end{array}$} \\
\hline & & $(+)$ & $(-)$ & \\
\hline \multirow[t]{2}{*}{ Glossalgia } & $(+)$ & 14 & 3 & $<0.01$ \\
\hline & $(-)$ & 90 & 93 & \\
\hline \multirow[t]{2}{*}{ Taste disorder } & $(+)$ & 10 & 4 & 0.13 \\
\hline & $(-)$ & 94 & 92 & \\
\hline \multirow[t]{2}{*}{ Dry mouth } & $(+)$ & 44 & 13 & $<0.01$ \\
\hline & $(-)$ & 60 & 83 & \\
\hline \multirow[t]{2}{*}{ Redness of oral mucosa } & $(+)$ & 8 & 1 & $<0.05$ \\
\hline & $(-)$ & 96 & 95 & \\
\hline \multirow[t]{2}{*}{ Redness of the tongue } & $(+)$ & 31 & 17 & $<0.05$ \\
\hline & $(-)$ & 73 & 79 & \\
\hline \multirow[t]{2}{*}{ Coated tongue } & $(+)$ & 47 & 23 & $<0.01$ \\
\hline & $(-)$ & 57 & 73 & \\
\hline \multirow[t]{2}{*}{ Angular cheilitis } & $(+)$ & 2 & 1 & 0.60 \\
\hline & $(-)$ & 102 & 95 & \\
\hline \multirow[t]{2}{*}{ Ulceration } & $(+)$ & 7 & 8 & 0.66 \\
\hline & $(-)$ & 97 & 88 & \\
\hline \multirow[t]{2}{*}{ Residual root } & $(+)$ & 27 & 3 & $<0.01$ \\
\hline & $(-)$ & 77 & 93 & \\
\hline \multirow[t]{2}{*}{ Denture } & $(+)$ & 36 & 7 & $<0.01$ \\
\hline & $(-)$ & 68 & 89 & \\
\hline
\end{tabular}

method showed the same sensitivity as the rinse method when high numbers of colonies were present; however, the concentrated rinse method was more sensitive when only a few colonies could be obtained from the sample. For the first 10 outpatients examined in this study, the

Table 6 Indices of clinical oral signs and detection of Candida by the concentrated rinse method

\begin{tabular}{|c|c|c|c|}
\hline \multicolumn{4}{|c|}{$\begin{array}{l}\text { Sensitivity, specificity, and positive predictive value between clinical oral } \\
\text { signs and detection of Candida by the concentrated rinse method }\end{array}$} \\
\hline Clinical oral signs & Sensitivity & Specificity & Positive predictive value \\
\hline Glossalgia & $13.5 \%$ & $96.9 \%$ & $82.4 \%$ \\
\hline Taste disorder & $9.6 \%$ & $95.8 \%$ & $71.4 \%$ \\
\hline Dry mouth & $42.3 \%$ & $86.5 \%$ & $77.2 \%$ \\
\hline Redness of oral mucosa & $7.7 \%$ & $99.0 \%$ & $88.9 \%$ \\
\hline Redness of the tongue & $29.8 \%$ & $82.3 \%$ & $64.6 \%$ \\
\hline Coated tongue & $45.2 \%$ & $76.0 \%$ & $67.1 \%$ \\
\hline Angular cheilitis & $1.9 \%$ & $99.0 \%$ & $66.7 \%$ \\
\hline Ulceration & $6.7 \%$ & $91.7 \%$ & $46.7 \%$ \\
\hline Residual root & $26.0 \%$ & $96.9 \%$ & $90.0 \%$ \\
\hline Denture & $34.6 \%$ & $92.7 \%$ & $83.7 \%$ \\
\hline
\end{tabular}


Table 7 Differences between the grades of each clinical oral sign and Candida numbers by the swab method

\begin{tabular}{|c|c|c|c|c|c|c|}
\hline \multirow[t]{2}{*}{ Clinical oral signs } & & \multicolumn{4}{|c|}{ Grade } & \multirow[b]{2}{*}{$p^{*}$} \\
\hline & & $\overline{0}$ & 1 & 2 & 3 & \\
\hline \multirow[t]{2}{*}{ Glossalgia } & Median $^{a}$ & 0 & 1 & 0 & 6.5 & 0.068 \\
\hline & $(n)$ & 183 & 9 & 6 & 2 & \\
\hline \multirow[t]{2}{*}{ Taste disorder } & Median & 0 & 0 & 0 & 116 & 0.282 \\
\hline & $(n)$ & 186 & 9 & 4 & 1 & \\
\hline \multirow[t]{2}{*}{ Dry mouth } & Median & 0 & 0.5 & 6 & 98 & $<0.001$ \\
\hline & $(n)$ & 143 & 36 & 20 & 1 & \\
\hline \multirow[t]{2}{*}{ Redness of oral mucosa } & Median & 0 & 24 & 0 & 0 & 0.015 \\
\hline & $(n)$ & 191 & 8 & 1 & 0 & \\
\hline \multirow[t]{2}{*}{ Redness of the tongue } & Median & 0 & 0 & 36.5 & 0 & 0.002 \\
\hline & $(n)$ & 152 & 40 & 8 & 0 & \\
\hline \multirow[t]{2}{*}{ Coated tongue } & Median & 0 & 0 & 0 & 7 & 0.138 \\
\hline & $(n)$ & 130 & 57 & 12 & 1 & \\
\hline \multirow[t]{2}{*}{ Angular cheilitis } & Median & 0 & 58 & 3 & $N A^{b}$ & 0.402 \\
\hline & $(n)$ & 197 & 2 & 1 & NA & \\
\hline \multirow[t]{2}{*}{ Ulceration } & Median & 0 & 0 & 0 & NA & 0.995 \\
\hline & $(n)$ & 185 & 11 & 4 & NA & \\
\hline \multirow[t]{2}{*}{ Residual root } & Median & 0 & 6 & 5 & NA & $<0.001$ \\
\hline & $(n)$ & 170 & 15 & 15 & NA & \\
\hline \multirow[t]{2}{*}{ Denture } & Median & 0 & 0 & 5 & NA & $<0.001$ \\
\hline & $(n)$ & 157 & 14 & 29 & NA & \\
\hline
\end{tabular}

*Kruskal-Wallis test

${ }^{a}$ Median of Candida numbers for every grade in a clinical oral sign

${ }^{\mathrm{b}} \mathrm{NA}$ not applicable

concentrated rinse method yielded more Candida colonies than the standard rise method, and the concentrated rinse method might generally show a higher sensitivity for detecting Candida in the oral cavity than the standard rinse method; therefore, we used results obtained via the concentrated rinse method rather than the standard rinse method for comparisons in the current study. Several sampling methods are available, including imprints, oral rinses, swabs, whole saliva collection [18], biopsies, and smears, and each method has both advantages and disadvantages [5]. Although the concentrated rinse method does not detect the localized site of infection, it enables quantitation of other microbes in addition to Candida species [5]. The concentrated rinse method is also easy to perform and is more sensitive than the imprint culture technique. Hence, it is suggested that the concentrated rinse method be preferentially employed in future investigations to obtain comparable data from different centers [8].

Candida counts may correspond to the severity of several clinical findings. Dry mouth was observed in 44 of 104 patients for whom Candida was detected by the concentrated rinse method, and the sensitivity, specificity,
Table 8 Differences between the grades of each clinical oral sign and Candida concentrations by the concentrated rinse method

\begin{tabular}{|c|c|c|c|c|c|c|}
\hline \multirow[t]{2}{*}{ Clinical oral signs } & & \multicolumn{4}{|c|}{ Grade } & \multirow[b]{2}{*}{$P^{*}$} \\
\hline & & $\overline{0}$ & 1 & 2 & 3 & \\
\hline \multirow[t]{2}{*}{ Glossalgia } & Median $^{a}$ & 0 & 137 & 1.5 & 1500.5 & 0.004 \\
\hline & (n) & 183 & 9 & 6 & 2 & \\
\hline \multirow[t]{2}{*}{ Taste disorder } & Median & 1 & 4 & 142.5 & 10000 & 0.163 \\
\hline & (n) & 186 & 9 & 4 & 1 & \\
\hline \multirow[t]{2}{*}{ Dry mouth } & Median & 0 & 127 & 269.5 & 1592 & $<0.001$ \\
\hline & (n) & 143 & 36 & 20 & 1 & \\
\hline \multirow[t]{2}{*}{ Redness of oral mucosa } & Median & 1 & 1186 & 121 & 0 & 0.008 \\
\hline & (n) & 191 & 8 & 1 & 0 & \\
\hline \multirow[t]{2}{*}{ Redness of the tongue } & Median & 0 & 3 & 791 & 0 & 0.006 \\
\hline & (n) & 152 & 40 & 8 & 0 & \\
\hline \multirow[t]{2}{*}{ Coated tongue } & Median & 0 & 7 & 22 & 1520 & 0.037 \\
\hline & (n) & 130 & 57 & 12 & 1 & \\
\hline \multirow[t]{2}{*}{ Angular cheilitis } & Median & 1 & 5000 & 578 & $N A^{b}$ & 0.417 \\
\hline & (n) & 197 & 2 & 1 & NA & \\
\hline \multirow[t]{2}{*}{ Ulceration } & Median & 1 & 1 & 0 & NA & 0.710 \\
\hline & (n) & 185 & 11 & 4 & NA & \\
\hline \multirow[t]{2}{*}{ Residual root } & Median & 0 & 275 & 254 & NA & $<0.001$ \\
\hline & (n) & 170 & 15 & 15 & NA & \\
\hline \multirow[t]{2}{*}{ Denture } & Median & 0 & 3 & 578 & NA & $<0.001$ \\
\hline & (n) & 157 & 14 & 29 & NA & \\
\hline
\end{tabular}

*Kruskal-Wallis test

${ }^{a}$ Median of Candida concentrations for every grade in a clinical oral sign ${ }^{\mathrm{b}} \mathrm{NA}$ not applicable

and positive predictive values of this characteristic were 42.3 , 86.5, and $77.2 \%$, respectively. The Candida concentrations obtained using the concentrated rinse method showed some significant differences in the severity of dry mouth, redness of the tongue, residual root, coated tongue, and denture.

Similarly, the absence of a number of clinical signs (oral mucosa redness, angular cheilitis, residual root, glossalgia, taste disorder, denture, and ulceration) was a robust indicator for the absence of Candida. Similarly, low densities of Candida may not cause coated tongue, dry mouth, denture, redness of the tongue, and residual root, which are often observed in outpatients with Candida in the oral cavity; indeed, the Candida density showed a significant difference between the severities of each of these signs.

Taste disorder, redness of the oral mucosa, angular cheilitis, and ulceration were observed in less than $10 \%$ of the outpatients diagnosed with candidiasis using the concentrated rinse method, and glossalgia was noted in $13.5 \%$ of the outpatients diagnosed with candidiasis using the concentrated rinse method. In any case, all the 
above clinical oral signs were likely to be related to other oral diseases rather than to Candida infection. Concentrations of less than $90 \mathrm{CFU} / 100 \mu \mathrm{L}$ obtained with the concentrated rinse method were not associated with any oral signs of candidiasis in outpatients and volunteers. The patients showing Candida colony numbers under $90 \mathrm{CFU} / 100 \mu \mathrm{L}$ in the concentrated rinse method might have been in the stage before apparent candidiasis.

Candida species are often detected in the oral cavity in healthy individuals, and their presence does not necessarily indicate Candida infection. A threshold Candida concentration is required in order to separate individuals with commensal Candida from those with infection-associated Candida. Most healthy Thai adolescents carry Candida at a low level, that is, below $50 \mathrm{CFU} / 100 \mu \mathrm{L}$ [19], and Candida levels of $60 \mathrm{CFU} / 100 \mu \mathrm{L}$ in concentrated rinse culture samples are associated with healthy commensal carriage [8]. On the other hand, individuals with conditions that predispose them to infection harbor higher numbers $\left(2 \times 10^{2}\right.$ to $\left.3 \times 10^{2} \mathrm{CFU} / 100 \mu \mathrm{L}\right)$. Candida levels up to $9 \times 10^{2} \mathrm{CFU} / 100 \mu \mathrm{L}$ have been observed in healthy controls without clinical oral signs in other studies [7, 9].

Quantitative analysis may be important for the assessment of oral candidiasis, including differentiation from the commensal carriage of Candida. Oral candidiasis is a particularly significant problem with respect to the morbidity of immunocompromised individuals, including HIV-positive and AIDS patients, organ transplant recipients, and chemotherapy patients $[10,20,21]$. In addition, there have been several reports on the relationships between oral Candida and diabetes mellitus [22], oral Candida and Sjögren's syndrome [23], and oral Candida and a combination of chronic renal failure and hemodialysis [24].

\section{Conclusions}

In this study, the Candida concentration associated with several clinical oral signs in the infected patients and may be closely related to the patient's current clinical status and prognosis. We have shown that quantitative analysis of Candida is required in order to correctly differentiate commensal forms of infection from those requiring treatment due to Candida infection. Such analysis may also be suitable for monitoring the time-dependent changes and quantitative analysis of Candida concentration. Adoption of the concentrated rinse method in independent locations around the globe is relatively straightforward since the method is simple. This will greatly facilitate direct comparisons between studies on Candida that originate in distinct geographic locations and involve diverse subject populations.

Abbreviations

CFU: colony forming unit; SD: standard deviation.

\section{Competing interests}

The authors declare that they have no competing interests.

\section{Authors' contributions}

HT performed the experiments and wrote the manuscript; TM contributed to planning and designing the study; $\mathrm{KH}, \mathrm{KK}$ and EK helped in the data collection; MU performed all the statistical analyses; HK and TH corrected the paper and supervised the study. All authors read and approved the final manuscript.

\section{Acknowledgements}

The authors wish to thank the participating patients and staff of the Department of Dentistry and Oral Surgery, Shinshu University School of Medicine, Shinshu University Hospital and the Department of Dentistry and Oral Surgery, Aizawa Hospital for their support in this study.

\section{Author details}

${ }^{1}$ Department of Dentistry and Oral Surgery, Shinshu University School of Medicine, Shinshu University Hospital, 3-1-1 Asahi, Matsumoto 390-8621, Japan. ${ }^{2}$ Department of Laboratory Medicine, Shinshu University School of Medicine, Shinshu University Hospital, 3-1-1 Asahi, Matsumoto 390-8621, Japan. ${ }^{3}$ Department of Dentistry and Oral Surgery, Aizawa Hospital, 2-5-1 Honjo, Matsumoto 390-8510, Japan. ${ }^{4}$ Center for Health, Safety and Environmental Management, Shinshu University, 3-1-1 Asahi, Matsumoto 390-8621, Japan.

Received: 10 May 2015 Accepted: 18 November 2015

Published online: 24 November 2015

\section{References}

1. Axéll T, Samaranayake LP, Reichart PA, Olsen I. A proposal for reclassification of oral candidosis. Oral Surg Oral Med Oral Pathol Oral Radiol Endod. 1997;84:111-2

2. Ghannoum MA, Jurevic RJ, Mukherjee PK, Cui F, Sikaroodi M, Naqvi A, et al. Characterization of the oral fungal microbiome (mycobiome) in healthy individuals. PLoS Pathog. 2010;8(6):e1000713. doi:10.1371/journal.ppat.1000713.

3. Zegarelli DJ. Fungal infections of the oral cavity. Otolaryngol Clin North Am. 1993;26:1069-89.

4. Sedgley CM, Samaranayake LP. The oral prevalence of aerobic and facultatively anaerobic gram-negative rods and yeasts in Hong Kong Chinese. Arch Oral Biol. 1994;39:459-66.

5. Williams DW, Lewis MA. Isolation and identification of Candida from the oral cavity. Oral Dis. 2000;6:3-11.

6. Byadarahally Raju S, Rajappa S. Isolation and identification of Candida from the oral cavity. ISRN Dent. 2011;2011:487921. doi:10.5402/2011/487921.

7. Samaranayake LP, MacFarlane TW, Lamey PJ, Ferguson MM. A comparison of oral rinse and imprint sampling techniques for the detection of yeast, coliform and Staphylococcus aureus carriage in the oral cavity. J Oral Pathol. 1986;15:386-8.

8. McKendrick AJ, Wilson MI, Main DM. Oral Candida and long-term tetracycline therapy. Arch Oral Biol. 1967;12:281-90.

9. White PL, Williams DW, Kuriyama T, Samad SA, Lewis MA, Barnes RA. Detection of Candida in concentrated oral rinse cultures by real-time PCR. J Clin Microbiol. 2004;42:2101-7.

10. Saunus JM, Kazoullis A, Farah CS. Cellular and molecular mechanisms of resistance to oral Candida albicans infections. Front Biosci. 2008;13:5345-58.

11. Hermann P, Berek Z, Nagy G, Kamotsay K, Rozgonyi F. Pathogenesis, microbiological and clinical aspects of oral candidiasis (candidosis). Acta Microbiol Immunol Hung. 2001;48:479-95.

12. Huskisson EC. Visual analogue scales. In: Merzack R, editor. Pain measurement and assessment. New York: Raven; 1983. p. 33-7.

13. Collins SL, Moore RA, McQuay HJ. The visual analogue pain intensity scale: what is moderate pain in millimeters? Pain. 1997;72:95-7.

14. Cancer Therapy Evaluation Program, Common Terminology Criteria for Adverse Events, Version 3.0, DCTD, NCl, NIH, DHHS, March 31, 2003, 2006, Aug 9. http:// ctep.cancer.gov.

15. Kakinoki Y, Nishihara T, Arita M, Shibuya K, Ishikawa M. Usefulness of new wetness tester for diagnosis of dry mouth in disabled patients. Gerodontology. 2004;21(4):229-31.

16. Eilers J, Berger A, Petersen M. Development, testing, and application of the oral assessment guide. Oncol Nurs Forum. 1988;15(3):325-30. 
17. Kojima K. Clinical studies on the coated tongue. Jpn J Oral Maxillofac Surg. 1985;31:45-64

18. Sugimoto J, Kanehira T, Mizugai H, Chiba I, Morita M. Relationship between salivary histatin 5 levels and Candida CFU counts in healthy elderly. Gerodontology. 2006;23:164-9.

19. Santiwongkarn P, Kachonboon S, Thanyasrisung P, Matangkasombut O. Prevalence of oral Candida carriage in Thai adolescents. J Investig Clin Dent. 2012;3:51-5.

20. Challacombe SJ, Naglik JR. The effects of HIV infection on oral mucosal immunity. Adv Dent Res. 2006;19:29-35.

21. Jain PA, Veerabhadrudu K, Kulkarni RD, Ajantha GS, Shubhada C, Amruthkishan U. Comparative study of adherence of oral Candida albicans isolates from HIV sero-positive individuals and HIV sero-negative individuals to human buccal epithelial cells. Indian J Pathol Microbiol. 2010;53:513-7.

22. Soysa NS, Samaranayake LP, Ellepola AN. Diabetes mellitus as a contributory factor in oral candidosis. Diabet Med. 2006;23:455-9.

23. Yan Z, Young AL, Hua H, Xu Y. Multiple oral Candida infections in patients with Sjogren's syndrome - prevalence and clinical and drug susceptibility profiles. J Rheumatol. 2011;38:2428-31.

24. Godoy JS, de Souza Bonfim-Mendonça P, Nakamura SS, Yamada SS, Shinobu-Mesquita C, Pieralisi N, et al. Colonization of the oral cavity by yeasts in patients with chronic renal failure undergoing hemodialysis. J Oral Pathol Med. 2013;42:229-34.

\section{Submit your next manuscript to BioMed Central and we will help you at every step:}

- We accept pre-submission inquiries

- Our selector tool helps you to find the most relevant journal

- We provide round the clock customer support

- Convenient online submission

- Thorough peer review

- Inclusion in PubMed and all major indexing services

- Maximum visibility for your research 